Abanico Veterinario. Enero-Diciembre 2020; 10:1-13. http://dx.doi.org/10.21929/abavet2020.18 Artículo Original. Recibido: 18/02/2020. Aceptado: 20/06/2020. Publicado: 14/08/2020.

\title{
Leucaena leucocephala y Opuntia ficus-indica reducen la producción de metano in vitro
}

\section{Leucaena leucocephala and Opuntia ficus-indica reduce the ruminal methane production in vitro}

\section{Araiza-Ponce Karina1 ID, Murillo-Ortiz Manuel ${ }^{1}$ ID, Herrera-Torres Esperanza ${ }^{2}$ ID, Valencia-Vázquez Roberto ${ }^{3}$ ID , Carrete-Carreón Francisco ${ }^{1 \text { ID }}$, Pámanes-Carrasco Gerardo*4 ID}

${ }^{1}$ Facultad de Medicina Veterinaria y Zootecnia. Universidad Juárez del Estado de Durango. Durango, México. C.P. 34307. ${ }^{2}$ Instituto Tecnológico del Valle del Guadiana. Tecnológico Nacional de México. Durango, México. ${ }^{3}$ Instituto Tecnológico de Durango. Tecnológico Nacional de México, Durango, México. C.P. 34080. ${ }^{4}$ Instituto de Silvicultura e Industria de la Madera. Universidad Juárez del Estado de Durango. Durango, México. *Autor de correspondencia: Pámanes-Carrasco Gerardo. Universidad Juárez del Estado de Durango. Blvd. Guadiana 501, Ciudad Universitaria. Durango, Durango, México. C.P. 34130. karii_araiza@hotmail.com, manuelmurillo906@gmail.com, heotes99@yahoo.com.mx, roberto.valenciav@gmail.com, focc1928mx@yahoo.com,gerardo.pamanes@gmail.com.

\section{RESUMEN}

El objetivo fue evaluar la inclusión de Leucaena leucocephala (LL) y Opuntia ficus-indica (OFI) fresco y fermentado como sustituto del heno de alfalfa $(\mathrm{HA})$ en la fracción forrajera sobre los patrones de fermentación, cinética de producción de gas y metano in vitro. Cuatro tratamientos (T1: $50 \%$ HA; T2: $30 \%$ LL y $20 \% \mathrm{HA}$; T3: $30 \%$ OFI y $20 \% \mathrm{HA}$; T4: $30 \%$ OFI fermentado y $20 \% \mathrm{HA}$ ) fueron formulados como dietas para bovinos. El contenido de compuestos fenólicos totales y taninos condensados se incrementaron más de $400 \%$ con T2 $(\mathrm{P}<0.05)$; los taninos condensados aumentaron $45 \%$ cuando se incluyó OFI fermentado en T4. La concentración de nitrógeno amoniacal, ácidos grasos volátiles, producción de gas y la relación acetato:propionato fueron diferentes entre tratamientos $(P<0.05)$. La máxima producción de gas se observó cuando se añadió $L L$ a la ración $(P<0.05)$; no se observaron cambios entre T1, T2 y T3 ( $P>0.05)$. Además, la fase lag disminuyó con T2 $(P<0.05)$. Adicionalmente, la producción de metano fue diferente entre tratamientos $(P<0.05)$; se observaron reducciones del $26 \%$ cuando se incluyó LL, y del $14 \%$ con OFI. De acuerdo con los resultados obtenidos, LL y el OFI son ingredientes que pudiesen incluirse en la dieta de bovinos y así, contribuir a la mitigación de gases de efecto invernadero.

Palabras clave: metanogénesis, Saccharomyces cerevisiae, fermentación ruminal, nopal forrajero.

\begin{abstract}
This study aimed to evaluate the inclusion of Leucaena leucocephala (LL) and Opuntia ficus-indica (OFI) fresh and fermented as alfalfa hay substitute in the forage fraction on the ruminal fermentative parameters, gas production kinetics and methane production in vitro. Four treatments were formulated as beef cattle diets (T1: $50 \%$ HA; T2: $30 \%$ LL y $20 \% \mathrm{HA}$; T3: $30 \%$ OFI y $20 \% \mathrm{HA}$; T4: $30 \%$ OFI fermentado y $20 \% \mathrm{HA}$ ). Total phenolics compounds and condensed tannins (CT) increased over $400 \%$ when $L L$ was included $(P<0.05)$. Additionally, CT increased $45 \%$ when fermented OFI was included in the ration in $\mathrm{T} 4(\mathrm{P}<0.05)$. Ammonia-nitrogen, volatile fatty acids, gas production and acetate:propionate ratio were different among treatments. The maximum gas production was observed when LL was included in T2 $(P<0.05)$. However, no changes were observed among T1, T2 and T3 $(P>0.05)$. In addition, lag phase decreased in T2 $(\mathrm{P}<0.05)$. Furthermore, methane production was different among treatments $(P<0.05)$; reductions of 26 and $14 \%$ were observed when including $L L$ and OFI, respectively. Thus, according to the obtained results, LL and OFI are ingredients, which may be included in the bovine diets and thereby contribute to the greenhouse gases mitigation.
\end{abstract}

Keywords: methanogenesis, Saccharomyces cerevisiae, ruminal fermentative, prickly pear. 


\section{INTRODUCCIÓN}

El sector ganadero ha sufrido transformaciones sustanciales en las últimas décadas. La creciente demanda resultante de la explosión demográfica exige incrementos en la producción ganadera (INECC, 2018). En México, la producción de bovinos ha tenido un crecimiento constante de $1.6 \%$ tan solo en la última década, con lo que se ostenta actualmente una producción de 1.88 millones de toneladas de carne en canal (INECC, 2018). No obstante, un incremento en la producción ganadera lleva consigo un aumento en las emisiones de gases de efecto invernadero (GEI), provenientes de la fermentación entérica ruminal; principalmente de metano. Además, la síntesis de metano ruminal le representa una pérdida de energía al animal de hasta $12 \%$ (Johnson y Johnson, 1995). El metano es un gas que presenta hasta 28 veces más poder calorífico que el $\mathrm{CO}_{2}$, por lo que se busca disminuir las emisiones del mismo. El metano antropogénico alcanza hasta $40 \%$ del total del metano emitido a la atmósfera, del cual $18 \%$ es atribuible a la ganadería a través de la fermentación entérica ruminal (IPCC, 2015). Tan solo en México, en 2015 se registró un total de emisiones de $510,043 \mathrm{Gg}$ de $\mathrm{CO}_{2} \mathrm{e}$ (equivalentes de $\mathrm{CO}_{2}$ ), de los cuales el 13\% está relacionado con la actividad ganadera; aproximadamente el $40 \%$ de estas emisiones es metano (INECC, 2018). Debido a esto, infinidad de investigaciones alrededor del mundo han tratado de crear diversas estrategias que disminuyan la producción de metano ruminal, a través de: modificaciones en la dieta, el empleo de diversos aditivos, vacunas, uso de extractos naturales, administración de compuestos químicos y, últimamente la suplementación de compuestos bio-activos extraídos de plantas (Martin et al., 2010; Pámanes-Carrasco et al., 2019).

Actualmente, los esfuerzos han sido enfocados a la utilización de fuentes de forraje no convencionales, que presenten una alternativa alimenticia en la producción animal. De este modo, algunas plantas cuyo uso no es común en la alimentación de rumiantes, pueden ser empleadas como una alternativa para reducir las emisiones de GEI. De hecho, debido a la limitación en la disponibilidad del forraje en las zonas áridas y semiáridas, hay algunos árboles, arbustos y cactáceas que pudieran emplearse como fuente de forraje. A este respecto, Leucaena leucocephala (LL) es conocida por tener una alta calidad nutricional, principalmente proteína (22-27\% MS; Aye y Adegun, 2013) y usualmente se adapta a la defoliación y rebrote en la época de secas; en zonas áridas puede llegar a presentar una producción de hasta 112 t ha ${ }^{-1}$ en zonas de hasta $430 \mathrm{~mm}$ de precipitación anual y una temperatura media de $32^{\circ} \mathrm{C}$ (Singh y Toky, 1995). Además, en investigaciones previas no se han reportado cambios en el consumo de materia seca y ganancia diaria de peso cuando se añadió $40 \%$ de LL a la ración (Piñeiro-Vázquez et al., 2017). De igual manera, ciertas cactáceas sin espinas, como la variedad de Opuntia ficus-indica (OFI), son consideradas forrajes frescos y palatables que se producen en la época seca, y representan una fuente importante de agua para los animales (González-Arreola et al., 2019). Sin embargo, el contenido de proteína en las cactáceas es bajo, por lo que los investigadores han desarrollado procedimientos biotecnológicos que mejoren el contenido de proteína 
cruda a través de fermentaciones en estado sólido, utilizando diferentes cultivos de levaduras de manera exitosa (Flores-Ortiz y Reveles-Hernández, 2010; Herrera et al., 2014, 2017). Adicionalmente, la adición de células vivas, como las levaduras, muestran una reducción en la metanogénesis en experimentos in vitro (Hristov et al., 2013). Además, ambas especies ( $L L$ y OFI) contienen metabolitos secundarios, tales como taninos condensados, saponinas y/o flavonoides, que pueden actuar como inhibidores de la síntesis de metano (Aye y Adegun, 2013; Alves et al., 2017; Pámanes-Carrasco et al., 2019;). Consecuentemente, el uso de estas especies como fuentes de forraje en la alimentación de rumiantes pueden ser una alternativa en la reducción de las emisiones de GEI, metano principalmente; sin afectar el desarrollo productivo.

Por lo tanto el objetivo del presente trabajo, fue evaluar la inclusión de Leucaena leucocephala y Opuntia ficus-indica fresco y fermentado, como sustituto del heno de alfalfa en la fracción forrajera sobre los patrones de fermentación, cinética de producción de gas y metano in vitro

\section{MATERIAL Y MÉTODOS}

\section{Área de estudio y materiales}

Esta investigación se llevó a cabo en el Laboratorio de Nutrición Animal de la Universidad Juárez del Estado de Durango, México. Las plantas de L. leucocephala fueron recolectadas al azar durante el otoño de 2017, de un cultivar ubicado en la misma Universidad. Las plantas colectadas midieron $1 \mathrm{~m}$ de longitud y el follaje fue removido manualmente después de la recolección. Las pencas de 0 . ficus-indica (variedad AV6) fueron colectadas de un plantío ubicado en terrenos aledaños a la Universidad. Los cultivos de levadura de Saccharomyces cerevisiae fueron adquiridos en un comercio local. En el cuadro 1 se muestra el análisis químico proximal de los ingredientes principales (alfalfa, LL, OFI y OFI fermentado).

\section{Fermentación de 0 . ficus-indica}

Las muestras de OFI fueron fermentadas con Saccharomyces cerevisiae a $32^{\circ} \mathrm{C}$, por $48 \mathrm{~h}$ de acuerdo a los procedimientos reportados por Herrera et al. (2014). Los cultivos de levadura se adicionaron al 1\% (MS). Después de la fermentación, las muestras fueron secadas y reducidas a un tamaño de partícula de $1 \mathrm{~mm}$ para su posterior análisis.

\section{Formulación de tratamientos experimentales y análisis químicos}

Cuatro tratamientos experimentales fueron formulados (cuadro 2), como dietas para ganado bovino con la inclusión de L. leucocephala (T2), O.ficus-indica (AV6), fresco (T3) y O. ficus-indica fermentado (T4), como sustitución parcial de la fracción de heno de alfalfa en el tratamiento testigo (T1). 
Cuadro 1. Caracterización química y nutricional de los ingredientes de la fracción forrajera en los tratamientos experimentales

\begin{tabular}{lcccc}
\hline & \multicolumn{4}{c}{ Ingredientes $(\%$ MS) } \\
\hline MS Parcial & Alfalfa & Leucaena & Nopal & Nopal Fermentado \\
MS Total & - & 44.9 & 9.3 & 8.3 \\
Materia orgánica & 89.7 & 89.5 & 90.0 & 88.1 \\
Proteína cruda & 87.1 & 91.5 & 72.0 & 79.6 \\
Extracto etéreo & 16.7 & 21.3 & 5.3 & 17.4 \\
FDN & 1.4 & 3.1 & 1.7 & 3.1 \\
FDA & 45.0 & 42.9 & 53.9 & 42.2 \\
Hemicelulosa & 27.6 & 13.8 & 13.5 & 21.2 \\
Celulosa & 17.3 & 29.1 & 40.3 & 21.0 \\
Lignina & 18.6 & 7.5 & 8.3 & 9.1 \\
DIVMS & 6.5 & 8.1 & 4.8 & 5.3 \\
DIVMO & 55.7 & 45.7 & 51.5 & 65.8 \\
CFT (mgEAG/gMS) & 50.0 & 42.3 & 40.0 & 59.8 \\
TC (mgEC/gMS) & 47.8 & 252.2 & 71.4 & 70.5 \\
mila seca; FDN: fibra & 5.3 & 69.3 & 3.1 & 2.0
\end{tabular}

MS: materia seca; FDN: fibra detergente neutra; FDA: fibra detergente acida; DIVMS: Digestibilidad in vitro de la materia seca a las 48h; DIVMO: Digestibilidad in vitro de la materia orgánica a las 48h; CFT: compuestos fenólicos totales; TC: taninos condensados; mgEAG: miligramos equivalentes de ácido gálico; mgEC: miligramos equivalentes de catequina.

Se prepararon $10 \mathrm{~kg}$ de cada tratamiento experimental y fueron mezclados en un mezclador rotativo con capacidad de 150 L (Gladiator PRO, modelo H8155/16); después, una muestra representativa de $1 \mathrm{~kg}$ fue tomada y mediante el método de cuarteo se tomaron sub-muestras para cada análisis. Todos los tratamientos experimentales fueron sujetos a análisis de composición química (cuadro 3) de acuerdo con los procedimientos estandarizados por AOAC (2010). Además, la fibra detergente neutro (FDN) y fibra detergente ácido (FDA), así como la celulosa, hemicelulosa y la fracción de lignina, fueron determinadas en un equipo Fiber Analyzer 200 (ANKOM Technology, USA), como lo propuso el fabricante (ANKOM, 2020). La digestibilidad in vitro de la material seca (DIVMS) y digestibilidad in vitro de la materia orgánica (DIVMO), fueron analizadas de acuerdo a los procedimientos sugeridos por ANKOM (2018).

Cuadro 2. Ingredientes de los tratamientos experimentales

\begin{tabular}{lcccc}
\hline & \multicolumn{5}{c}{ Tratamientos } \\
\hline Ingredientes (\% MS) & T1 & T2 & T3 & T4 \\
\hline Heno de alfalfa & 50 & 20 & 20 & 20 \\
L. leucocephala & 0 & 30 & 0 & 0 \\
O. ficus-indica & 0 & 0 & 30 & 0 \\
O. ficus-indica fermentado & 0 & 0 & 0 & 30 \\
Maíz molido & 30 & 30 & 30 & 30 \\
Harinolina & 19 & 19 & 19 & 19 \\
Mezcla de minerales & 1 & 1 & 1 & 1 \\
\hline
\end{tabular}




\section{Compuestos fenólicos totales y taninos condensados}

Los compuestos fenólicos totales (CFT), fueron determinados de acuerdo a los métodos propuestos por Heimler et al. (2005). Además, los taninos condensados (TC) fueron analizados como los reportó Porter et al. (1986).

\section{Patrones de fermentación in vitro}

Para los ensayos de fermentación in vitro, se obtuvo líquido ruminal de dos becerros de raza Brangus, dotados de cánula ruminal, con un peso aproximado de $450 \mathrm{~kg}$, y alimentados con ensilado de maíz y concentrado en una proporción 50:50. Aproximadamente, $1 \mathrm{~g}$ de muestra de cada tratamiento experimental fue incubado con $120 \mathrm{~mL}$ de solución buffer-inóculo ruminal en una relación $2: 1$ a $39^{\circ} \mathrm{C}$ en módulos de vidrio ANKOM (ANKOM Technology, USA), con tapas herméticas de goma y plástico, de acuerdo a lo propuesto por el fabricante por triplicado (ANKOM 2018). Después de transcurridas $24 \mathrm{~h}$ de incubación, los módulos fueron abiertos y el pH fue medido. Inmediatamente, se tomaron alícuotas de $10 \mathrm{~mL}$ para ser colocadas en frascos de vidrio, para posteriores análisis de ácidos grasos volátiles (AGV) y nitrógeno amoniacal, de acuerdo a lo propuesto por Galyean (2010).

\section{Producción de gas in vitro}

Aproximadamente $1 \mathrm{~g}$ de cada tratamiento experimental fue colocado en módulos de vidrio ANKOM (ANKOM Technologies, USA), equipados con un transductor de presión inalámbrico por triplicado. Las fermentaciones se llevaron de acuerdo a lo propuesto por el fabricante (ANKOM, 2018), incubando la muestra con una mezcla de solución buffer-inóculo ruminal en una relación 2:1. Las incubaciones se llevaron a cabo hasta las $96 \mathrm{~h}$ y los cambios de presión se registraron cada hora durante el proceso. La cinética de la producción de gas in vitro fue estimada mediante el ajuste de los datos obtenidos al modelo de Gompertz, de acuerdo a la siguiente ecuación (Murillo-Ortiz et al., 2018):

$$
G P=A e^{\left.-L e^{-(k} d\right)}
$$

En donde $\mathrm{GP}=$ producción de gas al tiempo $\mathrm{t}(\mathrm{mL}) ; A=$ máxima producción de gas $(\mathrm{mL}) ; \mathrm{k}_{\mathrm{d}}=$ constante de producción de gas $\left(\mathrm{h}^{-1}\right) ; \mathrm{y} \mathrm{L}=$ tiempo de latencia antes de que empiece la producción de gas (h). Adicionalmente, para las mediciones de las proporciones de metano y $\mathrm{CO}_{2}$, la válvula de alivio de presión de los módulos fue abierta por espacio de $2 \mathrm{~s}$, y el gas liberado fue conducido a través de un tubo hacia un analizador portátil de gas, de acuerdo al procedimiento propuesto por el fabricante (GEMTM5000, LANDTEC, USA) y adecuado por González-Arreola et al. (2019). La energía metabolizable (EM) fue estimada de acuerdo con la ecuación propuesta por Menke et al. (1979), la cual se presenta a continuación:

$$
E M=\left(1.1456 * G P_{24}\right)+(0.07675 * P C)+(0.1642 * E E)+1.198
$$

En donde EM= energía metabolizable (MJ/kg MS); GP24= producción de gas a las 24 h de incubación $(\mathrm{mL}) ; \mathrm{PC}=$ proteína cruda (\% MS); EE= extracto etéreo (\%MS). 


\section{Análisis estadístico}

Los datos experimentales obtenidos fueron analizados de acuerdo a un diseño completamente al azar, utilizando el procedimiento GLM del paquete estadístico SAS (2011). La comparación de medias se llevó a cabo mediante la prueba Tukey, declarando diferencias significativas con una $\mathrm{P}<0.05$.

\section{Cuadro 3. Análisis químico de los tratamientos experimentales}

\begin{tabular}{lcccc}
\hline \multicolumn{5}{c}{ Tratamientos } \\
\hline Nutrientes (\% MS) & T1 & T2 & T3 & T4 \\
\hline MO & 90.3 & 91.3 & 87.6 & 87.3 \\
PC & 17.4 & 18.1 & 14.3 & 17.5 \\
EE & 1.6 & 2.0 & 1.5 & 1.3 \\
FDN & 53.3 & 52.8 & 52.6 & 46.5 \\
FDA & 23.8 & 23.4 & 17.7 & 15.6 \\
Hemicelulosa & 29.5 & 29.4 & 34.9 & 30.9 \\
Celulosa & 16.3 & 14.5 & 11.9 & 11.6 \\
Lignina & 7.4 & 8.8 & 5.8 & 3.9 \\
DIVMO & 53.7 & 47.6 & 59.8 & 59.4 \\
EM (Mcal/kgMS) & 4.7 & 4.4 & 4.7 & 4.6 \\
\hline
\end{tabular}

MO: materia orgánica; PC: proteína cruda; EE: extracto etéreo; FDN: fibra detergente neutra; FDA: fibra detergente acida; DIVMO: Digestibilidad in vitro de la materia orgánica a las 48h; EM: Energía metabolizable; $\mathrm{T} 1: 50 \%$ alfalfa $+50 \%$ concentrado; T2: $20 \%$ alfalfa $+30 \% \mathrm{LL}+50 \%$ concentrado; T3: $20 \%$ alfalfa $+30 \% \mathrm{OFI}+50 \%$ concentrado; $\mathrm{T} 4: 20 \%$ alfalfa $+30 \%$ OFI fermentado $+50 \%$ concentrado.

\section{RESULTADOS Y DISCUSIÓN}

Los patrones fermentativos de los tratamientos experimentales se presentan en el cuadro 4. La concentración de nitrógeno amoniacal fue diferente entre los tratamientos $(\mathrm{P}<0.05)$; el valor más alto se obtuvo con el tratamiento testigo $(\mathrm{T} 1)$. Así, al agregar LL y OFI a la ración, se observaron reducciones del 26.5, 28.7 y $18.4 \%$ en T2, T3 y T4, respectivamente, en comparación con T1. Además, los valores obtenidos de $\mathrm{N}-\mathrm{NH}_{3}$ en este estudio se encuentran dentro del rango óptimo (5 a $10 \mathrm{mg} / \mathrm{dL}$ ), el cual maximiza el consumo de materia seca y el empleo de materia orgánica en el rumen (Chandrasekharaiah et al., 2011). Igualmente, la concentración de ácidos grasos volátiles (AGV) y ácidos grasos volátiles totales (AGVT) presentaron diferencias entre tratamientos $(\mathrm{P}<0.05)$. De acuerdo a lo expuesto por Sutton et al. (2003), las concentraciones normales de ácido acético, propiónico y butírico en una fermentación ruminal son aproximadamente de 60, 20 y 15\%, respectivamente; no obstante, las cantidades reportadas en este estudio difieren de las reportadas anteriormente.

Las concentraciones más altas de ácidos grasos volátiles totales (AGVT), así como ácido acético, se presentaron en T3. Por el contrario, las concentraciones de ácido butírico y propiónico fueron menores en el T3. No obstante, cuando se lleva a cabo el cálculo de la concentración molar de cada ácido graso volátil, las cantidades de acetato, propionato y butirato son mayores en T3 que en los demás tratamientos. 
Estos cambios en la concentración de AGV pueden estar relacionados con el incremento en los contenidos de carbohidratos, como la hemicelulosa; debido a la adición de OFI fresco a la ración. Al respecto, Johnson y Johnson (1995) comentan que una disminución en la relación acetato:propionato representa un proceso de fermentación más eficiente y una reducción en las pérdidas de energía mediante la formación de productos finales de la misma. Lo anterior se puede observar mediante la disminución en la producción de propionato, cuando se incluye OFI fresco a T3.

Cuadro 4. Patrones de fermentación ruminal in vitro y concentración de compuestos fenólicos de los tratamientos experimentales

\begin{tabular}{cccccc}
\hline & \multicolumn{5}{c}{ Tratamientos } \\
\hline & $\mathrm{T} 1$ & $\mathrm{~T} 2$ & $\mathrm{~T} 3$ & $\mathrm{~T} 4$ & EEM \\
\hline $\mathrm{pH}$ & $6.9 \pm 0.01$ & $7.0 \pm 0.04$ & $6.9 \pm 0.01$ & $6.9 \pm 0.01$ & 0.05 \\
$\mathrm{~N}-\mathrm{NH3}(\mathrm{mg} / \mathrm{dL})$ & $13.6 \pm 0.62^{\mathrm{a}}$ & $10.0 \pm 0.17^{\mathrm{b}}$ & $9.7 \pm 0.04^{\mathrm{b}}$ & $11.1 \pm 0.22^{\mathrm{b}}$ & 0.34 \\
AGVT (mM) & $78.7 \pm 1.32^{\mathrm{b}}$ & $78.7 \pm 2.04^{\mathrm{b}}$ & $132.9 \pm 6.39^{\mathrm{a}}$ & $76.3 \pm 1.88^{\mathrm{b}}$ & 1.03 \\
& & & & & \\
Acético (\% AGVT) & $46.8 \pm 0.39^{\mathrm{b}}$ & $46.4 \pm 0.77^{\mathrm{b}}$ & $57.0 \pm 1.28^{\mathrm{a}}$ & $45.1 \pm 0.65^{\mathrm{b}}$ & 0.84 \\
Propiónico (\% AGVT) & $32.7 \pm 0.32^{\mathrm{a}}$ & $33.2 \pm 0.51^{\mathrm{a}}$ & $25.1 \pm 1.05^{\mathrm{b}}$ & $34.0 \pm 0.46^{\mathrm{a}}$ & 0.64 \\
& & & & \\
Butírico (\% AGVT) & $15.0 \pm 0.02^{\mathrm{a}}$ & $14.9 \pm 0.15^{\mathrm{a}}$ & $12.4 \pm 0.15^{\mathrm{b}}$ & $15.4 \pm 0.07^{\mathrm{a}}$ & 0.11 \\
& & & & & \\
Acetato:Propionato & $1.4 \pm 0.02^{\mathrm{b}}$ & $1.4 \pm 0.4^{\mathrm{b}}$ & $2.2 \pm 0.14^{\mathrm{a}}$ & $1.3 \pm 0.03^{\mathrm{b}}$ & 0.08 \\
CFT (mgEAG/gMS) & $14.1 \pm 0.43^{\mathrm{c}}$ & $77.1 \pm 1.04^{\mathrm{a}}$ & $20.9 \pm 0.25^{\mathrm{b}}$ & $20.1 \pm 0.43^{\mathrm{b}}$ & 0.61 \\
TC (mgEC/gMS) & $1.55 \pm 0.03^{\mathrm{b}}$ & $20.4 \pm 0.17^{\mathrm{a}}$ & $0.90 \pm 0.07^{\mathrm{c}}$ & $0.50 \pm 0.08^{\mathrm{c}}$ & 0.10 \\
\hline
\end{tabular}

abLetras diferentes en la misma fila indican diferencia significativa $(\mathrm{p}<0.05)$. EEM: Error Estándar de la diferencia entre medias; N-NH3= Concentración de nitrógeno amoniacal a las 24h de fermentación in vitro; AGVT= Ácidos Grasos Volátiles Totales; CFT= Compuestos fenólicos totales; $\mathrm{TC}=$ Taninos condensados; mgEAG: miligramos equivalentes de ácido gálico; mgEC: miligramos equivalentes de catequina.

Como se puede observar en el cuadro 1, hay una diferencia marcada en las concentraciones de compuestos fenólicos totales (CFT), y de taninos condensados (TC) entre los diferentes ingredientes de la fracción forrajera. Debido a esto, en el cuadro 4, se muestran diferencias entre tratamientos en CFT y TC $(P<0.05)$. Los contenidos de CFT se incrementaron más de cuatro veces cuando se incluyó LL a la ración en T2. De la misma manera, CFT se incrementaron cerca del 45\% cuando se incluyó OFI a T3. Karimi et al. (2013) observaron contenidos tres veces menores de CFT cuando compararon alfalfa con LL. Además, los contenidos de CFT en los cladodios de OFI dependen de la madurez de la planta; mismos que se reportaron en valores cercanos a $33 \mathrm{mg} / \mathrm{g}$ de MS (Figueroa-Pérez et al. 2016). De manera similar, los contenidos de TC son diferentes entre los tratamientos experimentales $(P<0.05)$; cuando se incluyó LL a T2; el contenido de TC se incrementó cerca de $20 \mathrm{mgEC} / \mathrm{g}$ MS. Estos resultados concuerdan con los reportados por Berard et al. (2011). Debido a que LL es considerada como una planta tanífera, los contenidos de CFT y TC deben ser más altos en T2 en comparación con los demás. Además, cuando se adicionó OFI a T3 y T4, se observó una reducción en la concentración de TC. Márquez y Suárez 
(2008) reportaron un contenido de TC en la alfalfa cercanos a $0.5 \mathrm{mg} / \mathrm{g} \mathrm{MS}$; mientras que en los cladodios de OFI las concentraciones eran menores.

Este estudio encontró el mismo efecto. A este respecto, los altos contenidos de CFT con la inclusión de OFI pueden atribuirse a otros compuestos fenólicos diferentes de los TC, tal como lo afirmaron Cardador-Martínez et al. (2011). Además, Koenig et al. (2018) afirmaron que la adición de extractos puros de TC superiores a 3.5\%, no afecta el consumo de materia seca en becerros de engorda.

Los parámetros de la cinética de producción de gas se presentan en el cuadro 5 . La máxima producción de gas "A" fue diferente entre tratamientos $(P<0.05)$. El valor de "A" registrado en T4 fue diferente al obtenido en T2, presentando una media de 138.4 $(\mathrm{mL} / \mathrm{g} \mathrm{MS})(\mathrm{P}<0.05)$; mientras que los valores en la fase lag $(\mathrm{L})$ disminuyeron cuando se incluyó LL en T2, cuando es comparado con T3 y T4 $(\mathrm{P}<0.05)$. De acuerdo a lo que dice Van Soest (1994), los microorganismos celulolíticos tardan menos tiempo en atravesar la pared celular del forraje con un menor contenido de lignina; sin embargo, este efecto no se observa en este estudio. Aparentemente, los incrementos en la fracción de celulosa en T3 y T4 conllevan a un incremento en la fase lag (L). Este efecto lo encontró también Grilli et al. (2015), cuando midieron la degradabilidad de la hemicelulosa en diversos forrajes; la hemicelulosa contenida en la alfalfa se degradó en menor tiempo que la contenida en otras especies forrajeras.

Cuadro 5. Parámetros de la cinética de producción de gas, metano y dióxido de carbono de fermentaciones ruminales in vitro

\begin{tabular}{cccccc}
\hline & \multicolumn{5}{c}{ Tratamientos } \\
\hline & $\mathrm{T} 1$ & $\mathrm{~T} 2$ & $\mathrm{~T} 3$ & $\mathrm{~T} 4$ & $\mathrm{EEM}$ \\
\hline $\mathrm{A}(\mathrm{mL} / \mathrm{g} \mathrm{MS})$ & $155.4 \pm 1.78^{\mathrm{ab}}$ & $162.9 \pm 9.06^{\mathrm{a}}$ & $142.6 \pm 0.14^{\mathrm{ab}}$ & $138.4 \pm 3.14^{\mathrm{b}}$ & 1.65 \\
$\mathrm{kd}_{\mathrm{d}}\left(\mathrm{h}^{-1}\right)$ & $0.1 \pm 0.02$ & $0.1 \pm 0.01$ & $0.1 \pm 0.01$ & $0.1 \pm 0.01$ & 0.05 \\
$\mathrm{~L}(\mathrm{~h})$ & $2.0 \pm 0.15^{\mathrm{ab}}$ & $1.6 \pm 0.14^{\mathrm{b}}$ & $2.3 \pm 0.01^{\mathrm{a}}$ & $2.4 \pm 0.04^{\mathrm{a}}$ & 0.10 \\
$\mathrm{PG}_{24 \mathrm{~h}}(\mathrm{~mL} / \mathrm{gMS})$ & $123.7 \pm 6.30$ & $111.2 \pm 0.22$ & $122.0 \pm 0.56$ & $120.0 \pm 1.01$ & 1.31 \\
$\mathrm{CH}_{4}(\mathrm{~mL} / \mathrm{gMS})$ & $15.0 \pm 0.65^{\mathrm{a}}$ & $11.1 \pm 0.08^{\mathrm{c}}$ & $13.1 \pm 0.27^{\mathrm{b}}$ & $12.9 \pm 0.07^{\mathrm{b}}$ & 0.35 \\
$\mathrm{CO}_{2}(\mathrm{~mL} / \mathrm{gMS})$ & $102.8 \pm 3.85^{\mathrm{a}}$ & $90.5 \pm 0.39^{\mathrm{b}}$ & $98.9 \pm 2.32^{\mathrm{ab}}$ & $93.7 \pm 0.24^{\mathrm{ab}}$ & 1.22 \\
$\mathrm{CO}_{2}: \mathrm{CH}_{4}$ & $6.8 \pm 0.04^{\mathrm{c}}$ & $8.1 \pm 0.09^{\mathrm{a}}$ & $7.5 \pm 0.02^{\mathrm{b}}$ & $7.2 \pm 0.06^{\mathrm{b}}$ & 0.05 \\
\hline
\end{tabular}

abLetras diferentes en la misma fila indican diferencia significativa $(p<0.05)$. EEM: Error Estándar de la diferencia entre medias; $A=$ máxima producción de gas $(\mathrm{mL} / \mathrm{g} \mathrm{MS}) ; \mathrm{k}_{d}=$ Tasa constante de producción de gas $(\% / h) ; L=$ Fase de latencia $(\mathrm{h})$. T1: $50 \%$ alfalfa $+50 \%$ concentrado; T2: $20 \%$ alfalfa $+30 \%$ leucaena $+50 \%$ concentrado; T3: $20 \%$ alfalfa $+30 \%$ nopal $+50 \%$ concentrado; T4: $20 \%$ alfalfa $+30 \%$ nopal fermentado $+50 \%$ concentrado.

Por otro lado, la producción de gas a las $24 \mathrm{~h}$ no mostró cambios entre tratamientos (P>0.05). No obstante, se observaron reducciones del 26 y 14\% en la producción de metano con la inclusión de LL y OFI en T2 y T3, respectivamente; de los cuales T2 registró la menor producción de metano $(\mathrm{P}<0.05)$. Las reducciones en la producción de metano se pueden atribuir a la presencia de metabolitos secundarios en la ración. En este sentido, las más altas concentraciones de CFT y TC se presentaron en T2. Estos resultados concuerdan con los reportados por Tavendale et al. (2005), quienes establecen que los más altos contenidos de TC tienden a formar complejos con las proteínas, lo cual limita la degradación de la misma por acción de los microorganismos 
presentes durante la fermentación. Adicionalmente, la producción de metano disminuye mediante la reducción en la digestión de la fracción fibrosa. Esto último se observó en el T2, el cual presentó menor digestibilidad. De la misma manera, Tan et al. (2011) reportaron que los TC contenidos en LL pueden reducir la población de microorganismos metanogénicos, debido a la mayor presencia de protozoarios, y de esta manera afectar la transferencia de iones hidrógeno. Así, la producción de metano como captador de electrones se ve limitada. Además, la reducción en la producción de metano en T3 y T4 está directamente relacionada con la presencia de CFT. Lo anterior coincide con la investigación previa llevada a cabo por Tavendale et al. (2005) y Murillo-Ortiz et al. (2018). Estos autores afirman que la presencia de metabolitos secundarios como los CFT, son moléculas solubles y no fermentables que se encuentran negativamente relacionadas con la síntesis de metano.

Por su lado, la menor producción de $\mathrm{CO}_{2}$ se registró en T2, en comparación con T1 $(\mathrm{P}<0.05)$. Por el contrario, la mayor relación $\mathrm{CO}_{2}: \mathrm{CH}_{4}$ se encontró en T2 comparada con T1. Estos resultados son consistentes con la presencia de taninos condensados; los TC de las plantas reducen la metanogénesis ruminal mediante la inhibición de los metanogénicos (Tavendale et al., 2005). Asimismo, los altos valores en la relación $\mathrm{CO}_{2}: \mathrm{CH}_{4}$ en $\mathrm{T} 1$, indican que en algún punto de la ruta metábolica se está inhibiendo la metanogénesis, ya que hay más volumen de $\mathrm{CO}_{2}$ presente, mismo que no es sintetizado a metano.

La síntesis ruminal de metano involucra la reducción sucesiva de $\mathrm{CO}_{2}$ a metano, a través de diferentes niveles de formil, metileno y metil, y su reacción con las coenzimas; entre ellas la Coenzima $\mathrm{M}$ en el último paso de la síntesis (Liu y Whitman, 2008). A este respecto, Patra y Saxena (2010) mencionan que la actividad antimetanogénica de los taninos pudiera radicar en la actividad sobre ciertas proteínas funcionales (enzimas), localizadas en ciertos sitios accesibles de los matanógenos. Esta acción pudiera interrumpir la metanogénesis ruminal, mostrando la inhibición de $\mathrm{CH}_{4}$ en un punto donde el $\mathrm{CO}_{2}$ es consumido como sustrato; Murillo-Ortiz et al. (2018) observaron el mismo efecto.

\section{CONCLUSIÓN}

Estos resultados indican que la sustitución de alfalfa por Leucaena leucocephala, mantiene la calidad nutritiva; así como valores similares en la producción de gas y la cantidad de AGV in vitro. Adicionalmente, la inclusión del 30\% de Leucaena leucocephala y Opuntia ficus-indica reducen 26 y 14\% la concentración de metano in vitro, respectivamente. Por lo anterior, debido a su calidad nutricia y a sus características fermentativas pudiesen incluirse en la dieta de bovinos, y así contribuir a la mitigación de gases de efecto invernadero.

\section{LITERATURA CITADA}

ALMEIDA A, Nafarrete E, Alvarado A, Cervantes A, Luevanos Oropeza R, Balagurusamy N. 2011. Expresión genética en la digestión anaerobia: un paso adelante en la comprensión de las interacciones tróficas de esta biotecnología. Revista Científica de la Universidad Autónoma de Coahuila. 3(6):14-34. http://www.posgradoeinvestigacion.uadec.mx/AQM/No.\%206/3.html 
ALVES FAL, Andrade AP, Bruno RLA, Silva MGV, Souza MFV, dos Santos DC. 2017. Seasonal variability of phenolic compounds and antioxidant activity in prickly pear cladodes of Opuntia and Nopalea genres. Food Science and Technology. 37(4):536543. https://doi.org/10.1590/1678-457x.19316

ANKOM. 2018. RF Gas production system operator's manual. ANKOM Technology, USA. https://www.ankom.com/sites/default/files/document-files/RF_Manual.pdf

ANKOM. 2020. Fiber analyzer operator's manual. ANKOM Technology, USA. https://www.ankom.com/sites/default/files/document-files/A200_Manual.pdf

AOAC. 2010. Official method of Analysis. 18th Ed $3^{\text {rd }}$ Revision. Washington DC, USA. Association of Officiating Analytical Chemists. 2590 p. ISBN: 9780935584820

AYE PA, Adegun MK. 2013. Chemical composition and some functional properties of Moringa, Leucaena and Gliricidia leaf meals. Agricultural and Biology Journal of North America. 4(1):71-77. https://doi.org/10.5251/abjna.2013.4.1.71.77

BERARD NC, Wang Y, Wittenberg KM, Krause DO, Coulman BE, McAllister TA, Ominski KH. 2011. Condensed tannin concentrations found in vegetative and mature forage legumes grown in western Canada. Canadian Journal of Plant Sciences. $91: 669-675$. https://doi.org/10.4141/cjps10153

CARDADOR-MARTÍNEZ A, Jiménes-Martínez C, Sandoval G. 2011. Revalorization of cactus pear (Opuntia spp) wastes as a source of antioxidants. Ciência e Tecnologia de Alimentos. 31(3):782-788. https://doi.org/10.1590/S0101-20612011000300036

CHANDRASEKHARAIAH M, Thulasi A, Suresh KP, Sampath KT. 2011. Rumen degradable nitrogen requirements for optimum microbial protein synthesis and nutrient utilization in sheep fed on finger millet straw (Eleucine coracana) based diet. Animal Feed Science and Technology. 163:130-135. https://doi.org/10.1016/j.anifeedsci.2010.10.015

FIGUEROA-PÉREZ M, Pérez-Ramírez I, Paredes-López O, Mondragón-Jacobo C, Reynoso-Camacho R. 2016. Phytochemical composition and in vitro analysis of nopal (O. ficus-indica) cladodes at different stages of maturity. International Journal of Food Properties. 21(1):1728-1742. https://doi.org/10.1080/10942912.2016.1206126

FLORES-ORTIZ M, Reveles-Hernández M. 2010. Producción de nopal forrajero de diferentes variedades y densidades de plantación. In: VIII Simposium-Taller Nacional y 1er Internacional "Producción y Aprovechamiento del Nopal". Nuevo León, México: RESPYN; 2010. pp. 198-210. http://respyn2.uanl.mx/especiales/2010/ee-052010/documentos/17.pdf

GALYEAN ML. 2010. Laboratory Procedures in Animal Nutrition Research. 13 ed, Lubbock. USA. https://www.depts.ttu.edu/afs/home/mgalyean/lab_man.pdf

GONZÁLEZ-ARREOLA A, Murillo-Ortiz M, Pámanes-Carrasco G, Reveles-Saucedo F, Herrera-Torres E. 2019. Nutritive quality and gas production of corn silage with the addition of fresh and fermented prickly pear cladodes. Journal of Animal \& Plant Sciences. $\quad 40(1):$ 6544-6553. https://m.elewa.org/Journals/wpcontent/uploads/2019/04/4.Gonzalez.pdf 
GRILLI DJ, Páez-Lama SA, Egea AV, Cerón-Cucchi ME, Cobos E, Allegretti L, Arenas N. 2015. Degradación y utilización de la hemicelulosa contenida en especies forrajeras Pseudobutyrivibrio ruminis y Pseudobutyrivibrio xilanivorans. REV FCA/Uncuyo. 47(2):231-243.

https://www.redalyc.org/pdf/3828/Resumenes/Resumen_382842590018_1.pdf

HEIMLER D, Vignolini P, Dini M, Romani A. 2005. Rapid Tests to Assess the Antioxidant Activity of Phaseolus vulgaris L. Dry Beans. Journal of Agriculture and Food Chemestry. 53(8):3053-3056. https://doi.org/10.1021/jf049001r

HERRERA-TORRES E, Murillo M, Berumen L, Páez J, Villarreal G. 2014. Efecto de Sacharomyces cerevisiae y Kluyveromices marxianus durante el tiempo de fermentación en la calidad nutritiva del nopal forrajero. Ecosistemas y Recursos Agropecuarios. 1(1):33-40. http://www.scielo.org.mx/pdf/era/v1n1/v1n1a4.pdf

HERRERA TE, Murillo M, Berumen L, Soto-Cruz NO, Páez-Lerma JB. 2017. Protein Enrichment of Opuntia Ficus-indica using Kluyveromyces marxianus in solid-state fermentation. Ciencia e Investigación Agraria. 44:113-120. http://dx.doi.org/10.7764/rcia.v44i2.1767

HRISTOV AN, Oh J, Firkins JL, Dijkastra J, Kebreab E, Waghorn G, Makkar HPS, Adesogan AT, Yang W, Lee C, Gerber PJ, Henderson B, Tricario JM. 2013. Special Topics - Mitigation of methane and nitrous oxide emissions from animal operations: I. A review of enteric methane mitigation options. Journal of Animal Science. 91:50455069. http://dx.doi.org/10.2527/jas.2013-6583

IPCC. 2015: Meeting Report of the Intergovernmental Panel on Climate Change Expert Meeting on Climate Change, Food, and Agriculture. Mastrandrea MD, Mach KJ, Barros VR, Bilir TE, Dokken DJ, Edenhofer O, Field CB, Hiraishi T, Kadner S, Krug T, Minx JC, PichsMadruga R, Plattner JK, Qin D, Sokona Y, Stocker TF, Tignor M (eds.). World Meteorological Organization, Geneva, Switzerland, 68 pp. https://www.ipcc.ch/publications_and_data/publications_and_data_supporting_materi al.shtmL

INECC. 2018. Sexta comunicación nacional y segundo reporte bienal de actualización ante la convención marco de las Naciones Unidas sobre el cambio climático. Secretaria del Medio ambiente y recursos naturales (SEMARNAT). Instituto Nacional de Ecología y Cambio Climático. México. http://cambioclimatico.gob.mx:8080/xmlui/handle/publicaciones/117

JOHNSON KA, Johnson DE. 1995. Methane emissions from cattle. Journal of Animal Science. 73:2483-2492. https://doi.org/10.2527/1995.7382483x

KARIMI E, Oskoueian E, Oskoueian A, Omidvar V, Hendra R, Nazeran H. 2013. Insight into the functional and medicinal properties of Medicago sativa (Alfalfa) leaves extract. Journal of Medical Plants Research. 7(7):290-297. https://doi.org/10.5897/JMPR11.1663 
KOENIG KM, Beauchemin KA, McGinn SM. 2018. Feeding condensed tannins to mitigate ammonia emissions from beef feedlot cattle fed high-protein finishing diets containing distillers grains. Journal of Animal Science. 96(10):4414-4430. https://doi.org/10.1093/jas/sky274

LIU Y, Whitman WB. 2008. Metabolic, Phylogenetic, and Ecological Diversity of the Methanogenic Archaea. Annals of the New York Academy of Sciences. 1125(1):171189. https://doi.org/10.1196/annals.1419.019

MÁRQUEZ D, Suárez A. 2008. El uso de taninos condensados como alternativa nutricional y sanitaria en rumiantes. Revista de Medicina Veterinaria. 16:87-109. https://doi.org/10.19052/mv.1449

MARTIN C, Morgavi DP, Doreau M. 2010. Methane mitigation in ruminants: from microbe to the farm scale. Animal. 4(3):351-365. https://doi.org/10.1017/S1751731109990620

MENKE K, Raab L, Salewski A, Steingass H, Fritz D, Schneider W. 1979. The estimation of the digestibility and metabolizable energy content of ruminant feedingstuffs from the gas production when they are incubated with rumen liquor in $\begin{array}{lll}\text { vitro. Journal of Agricultural Science. } & \text { 93(1):217-222. }\end{array}$ https://doi.org/10.1017/S0021859600086305

MURILLO OM, Herrera TE, Corral LA, Pámanes CG. 2018. Effect of inclusion of graded level of water hyacinth on in vitro gas production kinetics and chemical composition of alfalfa hay based beef cattle diets. Indian Journal of Animal Research. 52(8):1298-1303. https://doi.org/10.18805/ijar.11417

PÁMANES-CARRASCO G, Herrera-Torres E, Murillo-Ortiz M, Reyes-Jáquez D. 2019. "Climate change mitigation in livestock produciton: nonconventional feedstuffs and alternative additives". En: Abubakar M, Livestock health and farming. London, UK: IntechOpen publishers. http://doi.org/10.5772/intechopen.89433

PATRA AK, Saxena J. 2010. A new perspective on the use of plant secondary metabolites to inhibit methanogenesis in the rumen. Pshytochemistry. 71(11-12):11981222. http://doi.org/10.1016/j.phytochem.2010.05.010

PIÑEIRO-VÁZQUEZ AT, Jiménez-Ferrer GO, Chay-Canul AJ, Casanova-Lugo F, Días-Echeverría VF, Ayala-Burgos AJ, Solorio-Sánchez FJ, Aguilar-Pérez CF, KuVera JC. 2017. Intake, digestibility, nitrogen balance and energy utilization in heifers fed low-quality forage and Leucaena leucocephala. Animal Feed Science and Technology. 228:194-201. https://doi.org/10.1016/j.anifeedsci.2017.04.009

PORTER LJ, Hrstich LN, Chan BG. 1986. The conversion of procyanidins and prodelphinidins to cyanidin and delphinidin. Phytochemistry. 25:223-230. https://doi.org/10.1016/S0031-9422(00)94533-3

SAS Institute Inc. 2011. SAS 9.3. Cary, NC: SAS Institute Inc. ISBN 978-1-60764-8963 
SUTTON JD, Dhanoa MS, Morant SV, France J, Napper DJ, Schuller E. 2003. Rates of Production of Acetate, Propionate, and Butyrate in the Rumen of Lactating Dairy Cows Given Normal and Low-Roughage Diets. Journal of Dairy Science. 86:36203633. https://doi.org/10.3168/jds.S0022-0302(03)73968-X

SINGH V, Toky OP. 1995. Biomass and net primary productivity in Leucaena, Acacia and Eucalyptus, shor rotation, high density ('energy') plantations in arid India. Journal of Arid Environments. 31(3):301-309. https://doi.org/10.1016/S0140-1963(05)80034-5

TAN HY, Sieo CC, Abdullah N, Liang JB, Huang XD, Ho YW. 2011. Effects of condensed tannins from Leucaena on methane production, rumen fermentation and populations of methanogens and protozoa in vitro. Animal Feed Science and Technology. 169(3-4):185-193. https://doi.org/10.1016/j.anifeedsci.2011.07.004

TAVENDALE MH, Meagher LP, Pacheco D, Walker N, Attwood GT, Sivakumaran S. 2005. Methane production from in vitro rumen incubations with Lotus pedunculatus and Medicago sativa, and effects of extractable condensed tannin fractions on methanogenesis. Animal Feed Science and Technology. 123:403-419. https://doi.org/10.1016/j.anifeedsci.2005.04.037

VAN SOEST PJ. 1994. Nutritional ecology of the ruminant. 2da Edición. Ithaca, EEUU. Cornell University Press. Pp. 476. ISBN: 9780801427725. 